Atmos. Chem. Phys., 13, 5243-5253, 2013

www.atmos-chem-phys.net/13/5243/2013/

doi:10.5194/acp-13-5243-2013

(c) Author(s) 2013. CC Attribution 3.0 License.

\title{
On the scaling effect in global surface air temperature anomalies
}

\author{
C. A. Varotsos $^{1}$, M. N. Efstathiou ${ }^{1}$, and A. P. Cracknell ${ }^{2}$ \\ ${ }^{1}$ Climate Research Group, Division of Environmental Physics and Meteorology, Faculty of Physics, University of Athens, \\ University Campus Bldg. Phys. V, Athens 15784, Greece \\ ${ }^{2}$ Division of Electronic Engineering and Physics, University of Dundee, Dundee DD1 4HN, Scotland, UK
}

Correspondence to: C. A. Varotsos (covar@phys.uoa.gr)

Received: 8 April 2012 - Published in Atmos. Chem. Phys. Discuss.: 8 June 2012

Revised: 29 April 2013 - Accepted: 2 May 2013 - Published: 27 May 2013

\begin{abstract}
The annual and the monthly mean values of the land-surface air temperature anomalies from 1880-2011, over both hemispheres, are used to investigate the existence of long-range correlations in their temporal evolution. The analytical tool employed is the detrended fluctuation analysis, which eliminates the noise of the non-stationarities that characterize the land-surface air temperature anomalies in both hemispheres. The reliability of the results obtained from this tool (e.g., power-law scaling) is investigated, especially for large scales, by using error bounds statistics, the autocorrelation function (e.g., rejection of its exponential decay) and the method of local slopes (e.g., their constancy in a sufficient range). The main finding is that deviations of one sign of the land-surface air temperature anomalies in both hemispheres are generally followed by deviations with the same sign at different time intervals. In other words, the land-surface air temperature anomalies exhibit persistent behaviour, i.e., deviations tend to keep the same sign. Taking into account our earlier study, according to which the land and sea surface temperature anomalies exhibit scaling behaviour in the Northern and Southern Hemisphere, we conclude that the difference between the scaling exponents mainly stems from the sea surface temperature, which exhibits a stronger memory in the Southern than in the Northern Hemisphere. Moreover, the variability of the scaling exponents of the annual mean values of the land-surface air temperature anomalies versus latitude shows an increasing trend from the low latitudes to polar regions, starting from the classical random walk (white noise) over the tropics. There is a gradual increase of the scaling exponent from low to high latitudes (which is stronger over the Southern Hemisphere).
\end{abstract}

\section{Introduction}

The land-surface air temperature (LSAT) is a crucial thermodynamic parameter of the atmosphere (which is a subsystem of the Earth's climate system), affected, among other things, by the atmospheric greenhouse effect. According to current scientific understanding, the spatial and temporal fluctuations of LSAT are generated by natural- and anthropogenicallyinduced mechanisms. The prediction of these mechanisms is of great importance for the description of the temporal evolution of various parameters of the other subsystems of the climatic system (i.e., the hydrosphere, lithosphere, cryosphere and biosphere), given that all the sub-systems of the climate system interact between them via non-linear processes (e.g., Anthis and Cracknell, 2005; Alexandris et al., 1999; Chandra and Varotsos, 1995; Efstathiou et al., 1998, 2003; Feretis et al., 2002; Katsambas et al., 1997; Kondratyev and Varotsos, 1995a, b, c, 1996; Melnikova, 2009; Tzanis and Varotsos, 2008; Varotsos et al., 1994; Xue et al., 2011). In other words, the temporal fluctuations of LSAT are of composite nature, consisting of periodic and non-periodic components (e.g., Chattopadhyay and Chattopadhyay, 2010; Efstathiou and Varotsos, 2010). These interactions may occur between different parts of the same subsystem. As an example, the stratosphere plays a substantial role in the natural and forced variability of the Earth system. In this regard, the lower stratosphere plays an important role in the radiative balance of the troposphere (two-way coupling), and additionally changes in greenhouse gases (GHGs), such as ozone and water vapour, in the lower stratosphere directly affect surface temperatures (e.g., Gerber et al., 2012).

It has been stated by Koscielny-Bunde et al. (1998) that the deviations of the daily maximum temperatures from their 
average values follow a universal scaling law (or persistence law) with roughly the same exponent, $a=0.7$. This behaviour was said to be found in the atmospheric variability by 14 meteorological stations around the globe, but in a later study Weber and Talkner (2001) found differences between these exponent values, depending on the altitude of the meteorological station.

In this regard, Eichner et al. (2003) studied the appearance of long-term persistence in surface air temperature records, obtained at 95 stations all over the globe using several variants of the detrended fluctuation analysis (DFA). They obtained correlation exponent values in the range $0.55-0.9$ centered strongly at around 0.65 over continents, but systematically higher values for islands.

The DFA tool was also used by Maraun et al. (2004) in order to study the existence of long-range correlations, which mathematically denote that the correlation sum is divergent. Maraun et al. (2004) demonstrated that if the autocorrelation function of a process decays according to a power law for large time lags, then it exhibits scaling, i.e., a long-memory process, which in fact refers to a non-periodic process. In addition, they claimed that power-law scaling of the fluctuation function cannot be regarded a priori, but it should be established in conjunction with the investigation of the local slopes. They argued that the comparison of a long-memory with a short-memory model does not specify the existence of long-range correlations from the application of DFA on a finite dataset. Moreover, they remarked that scaling cannot be concluded from a log-log straight line fit to the fluctuation function.

Recently, six reconstructed records of the Northern Hemisphere (NH) temperatures, analysed by Rybski et al. (2006), revealed long-term persistence. Due to the long-term persistent correlations, the mean temperature variations $\sigma(m$, $L$ ) between $L$ years (moving averages over $m$ years) were substantially larger than for uncorrelated or short-term correlated data.

Finally, in our previous study (Efstathiou et al., 2011) we studied the scaling behaviour of the time series of the hemispheric mean monthly land- and sea surface temperature (LSST) anomalies during the period January 1850August 2008. The datasets employed were obtained from the Climatic Research Unit (School of Environmental Sciences of University of East Anglia; www.cru.uea.ac.uk/cru/ data/temperature/). We showed that there is persistent powerlaw scaling in the time series of the LSST anomalies, which stems from the time evolution of their values and not from their marginal distribution. Remarkably, this persistency was found to be stronger in the Southern Hemisphere (SH) (see also Alvarez-Ramirez et al., 2008).

In the present paper, we substantially extend the abovementioned analysis by considering the LSAT anomalies for various latitudinal zones of each hemisphere separately and globally. Our principal aim is to investigate the intrinsic scaling properties of the LSAT anomalies and to compare them with those of LSST anomalies found in Efstathiou et al. (2011). In other words, we investigate whether the fluctuations of the LSAT anomalies in small time intervals are significantly correlated to those in longer time intervals and to identify with reasonable accuracy the type of this correlation. The robustness (or not) of the result obtained (or not) is critical to whether this can be exploited further. For instance, the current climate models do not display scaling behaviour, especially for the scenario of the climate change induced from the increased or even constant GHGs concentrations (e.g., Koutsoyiannis et al., 2008). Therefore, there is an urgent need for the development of such climate models, which should be based on robust evidence for scaling behaviour at long time scales. The main insight of this paper is the robustness of the long-memory evidence in LSAT, which had not been studied to date.

\section{Data and analysis}

For the above mentioned purposes we employed the annualand monthly mean values of LSAT anomalies (in 0.01 degrees Celsius) obtained from the National Aeronautics and Space Administration Goddard Institute for Space Studies, covering the period 1880-2011 (http://data.giss.nasa.gov/ gistemp/). As it is clearly stated on this website, the analysis is limited to the period since 1880 because of poor spatial coverage of stations and decreasing data quality prior to that time.

As discussed in the previous section, the main aim of this paper is to search efficiently for time scaling in the LSAT anomalies time series, by using an analytical tool that would not be affected by the non-stationarities of the time series, compared to the classical methods, like the autocorrelation function, Fourier analysis, etc. The technique that has been proved to satisfy this criterion is the well-established DFA, which stems from random walk theory and permits the detection of intrinsic self-similarity in non-stationary time series (Peng et al., 1994; Weber and Talkner, 2001; Varotsos, 2005a, b; Varotsos and Kirk-Davidoff, 2006; Varotsos et al., 2002, 2003, 2005, 2006, 2007, 2009).

The sequential steps of DFA may be briefly described as follows. Firstly, the $y(i)=$ LSAT anomalies time series is integrated and is divided into a series of non-overlapping boxes of equal length, $\tau$. Then in each box a best polynomial local trend (of order $l$ ) is fitted in order to detrend the integrated profile by subtracting the locally fitted trend. Secondly, for each box the root-mean-square fluctuation $F_{d}(\tau)$ is calculated. The squared fluctuation function of this integrated and detrended profile is calculated by averaging over all boxes. Thirdly, this procedure is repeated for several boxes' lengths. More specifically, the detrended fluctuation function $F$ is defined by Kantelhardt et al. (2002): 
$F^{2}(\tau)=\frac{1}{\tau} \sum_{i=k \tau+1}^{(k+1) \tau}[y(i)-z(i)]^{2}, k=0,1,2, \ldots,\left(\frac{N}{\tau}-1\right)$

where $z(i)$ is the polynomial of order $l$ least-square fit to the $\tau$ data points contained in a box. Due to natural variability, a reasonable choice for the maximum scale is about $1 / 10$ of the total record length (Maraun et al., 2004). In our analysis, below $F(\tau)$ is calculated for maximum scale $N / 4$ approximately.

It is worth noting that averaging $F(\tau)$ over the $N / \tau$ intervals gives the fluctuation $<F(\tau)>$ as a function of $\tau$. For scaling dynamics, the averaged $F^{2}(\tau)$ over the $N / \tau$ intervals with length $\tau$ is expected to obey a power law, notably

$<F^{2}(\tau)>\sim \tau^{2 \alpha}$

and the power spectrum function scales with $1 / f^{\beta}$, with $\beta=$ $2 a-1$ (Taqqu et al., 1995).

A derived DFA exponent $a \neq 1 / 2$ denotes the existence of power-law correlations for large scales, while $a=1 / 2$ reveals the classical random walk (white noise). If $0<\alpha<0.5$, power-law anticorrelations are present (antipersistence). If $0.5<\alpha<1.0$, then persistent power-law correlations prevail. The case $a=1$ corresponds to the so-called $1 / f$ noise (e.g., Hausdorff and Peng, 1996). In addition, when $1<\alpha<1.5$, then long-range correlations are present (Weber and Talkner, 2001). In other words, the DFA exponent could be assumed as a measure of the long-range dependence (LRD). A more detailed description of the DFA tool is presented by Efstathiou et al. (2011). It should be stressed that the power-law scaling deduced by the DFA fluctuation function must not be taken as evidence for long-range correlations. As mentioned above, in order to reliably infer power-law scaling, it must be established by investigating the constancy of local slopes in a sufficient range, rejecting also the exponential decay of the autocorrelation function (Maraun, et al., 2004; Rust, 2007; Tsonis and Elsner, 1995).

The reason we have used both annual mean and monthly mean temperature anomalies is due to the ongoing debate about the minimum allowable amount of data required to reliably employ the DFA methodology (Bryce and Sprague, 2012). Some of the earlier studies claim that DFA requires a large sample size (i.e., 1000+ data points), while others suggest a definitive minimum sample size (e.g., 600 by Ludecke et al., 2011, or less by Delignieres et al., 2006 and Cororando and Carpena, 2012). In particular, Coronado and Carpena (2005) studied the influence of the length of a time series on the results obtained from several techniques used to detect long-range correlations. They reached the conclusion that the DFA method is practically free of size effects, providing consistently correct results for all the ranges/blackbox[CE]Note that "range" was made plural for this sentence to make semantic sense. But you may have meant "for the entire range of time series lengths." Please check. of time series lengths. In this context, Delignieres et al. (2006) tested similar methods for the estimation of the scaling exponent, focussing in particular on short time series (i.e., series of 2048, 1024, 512, 256, 128 and 64 data points). According to the results presented by Delignieres et al. (2006), DFA worked particularly well with fractional Gaussian noise (fGn) series with no significant bias and no effect of data series length, whatever the true value of $\alpha$ exponent.

In addition, there are other methods which give error bounds, like spectral estimators (e.g., Geweke and PorterHudak, 1983), which claim to be superior to DFA. In a later study, Hurvich and Brodsky (2001) found that a few choices, originally suggested by Geweke and Porter-Hudak (1983), can lead to performance that is markedly inferior to that of the optimal choice, even in reasonably small samples. Very recently, Franzke et al. (2012) studied the performance of tools (among them DFA) that were originally developed to detect LRD, and have found application in the prediction of dangerous bifurcations in dynamical systems, such as climate "tipping points". The conclusion drawn by Franzke et al. (2012) is verbatim: "While it is true that all of the estimators we tested perform reasonably well for fractional Gaussian noise, once a time series is non-Gaussian or is nonstationary (in trend or volatility) the estimators can be problematic."

Under these circumstances one could argue that the annual temperature anomalies should not be used for DFA due to their limited sample size, and suggest that the monthly temperature anomalies should be utilised instead. Thus, we decided to analyse here both annual and monthly temperature anomalies, including the corresponding error estimates.

In more detail, the data analysed here refer to annual mean values of LSAT anomalies with 131 data points and 1572 data points for the monthly values of LSAT anomalies. It should be emphasized that Audit et al. (2002) demonstrated that the wavelet transform modulus maxima estimator leads to larger mean squared errors (compared to those obtained by DFA) when analysing short time series of length $10^{2}$ data points. That is why it was preferred to use DFA and not, for example, wavelet based estimators of self-similarity (Varotsos et al., 2007; see also the interactive discussion, i.e., SC S5324).

\section{Results and discussion}

\subsection{DFA exponent in the time series of the annual mean LSAT anomalies}

As mentioned above, the annual means used in the present study were based on LSAT anomalies covering the whole Earth and both hemispheres (Fig. 1). The principal features shown in Fig. 1 are the existence of non-stationarities in the LSAT anomalies and the strong upward trend that can both mask correlations and in particular long-term correlations. 

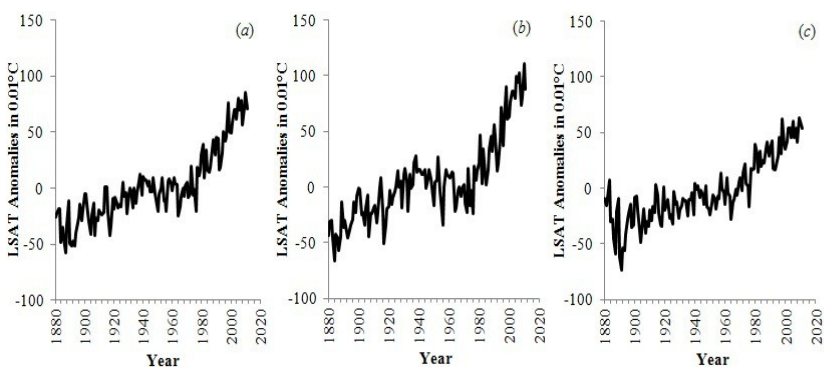

Fig. 1. Annual LSAT anomalies in $0.01^{\circ} \mathrm{C}$, during the period 1880 2011, (a) globally, (b) over the $\mathrm{NH}$ and (c) over the SH.
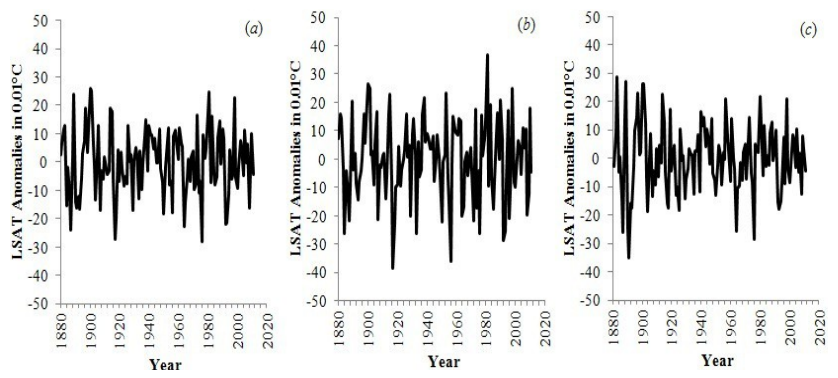

Fig. 2. Detrended annual mean LSAT anomalies in $0.01^{\circ} \mathrm{C}$, during the period 1880-2011, (a) globally, (b) over the NH and (c) over the $\mathrm{SH}$.

For instance, uncorrelated data with a long-term trend may look like correlated data. Also, long-term correlated data may look like uncorrelated data due to a trend. Thus, the longterm trend was filtered out by calculating the departures of the LSAT anomalies values from the polynomial best fit (of sixth order) to the whole LSAT anomalies time series, over the whole Earth and both hemispheres, separately (Fig. 2).

In the following, the results obtained from the application of DFA to the detrended LSAT anomalies time series are presented and interpreted. The DFA exponent for the detrended annual mean LSAT anomalies over the globe was found to be $a=0.68$ and over both hemispheres $a=0.65$, with standard deviation 0.03 for all geographical areas. Therefore, the fluctuations of the studied parameter exhibit long-range persistence for large scales up to $32 \mathrm{yr}$ (Fig. 3).

In order to examine whether the aforementioned value of the $a$-exponent is attributed to the temporal evolution of the LSAT anomalies and not from their marginal distribution, the investigated time series was randomly shuffled. If the shuffled LSAT anomalies values followed the random (white) noise, then the persistence found above would not come from the data but from their temporal evolution. In this regard, we applied the Monte Carlo method, relying on 1000 repeated random samplings of the detrended annual mean LSAT anomalies (over the NH, SH and globe, separately) to compute the corresponding $a$-exponents. According to the Kolmogorov-Smirnov (Stephens, 1974) and Anderson-Darling (Anderson and Darling, 1954) best fit
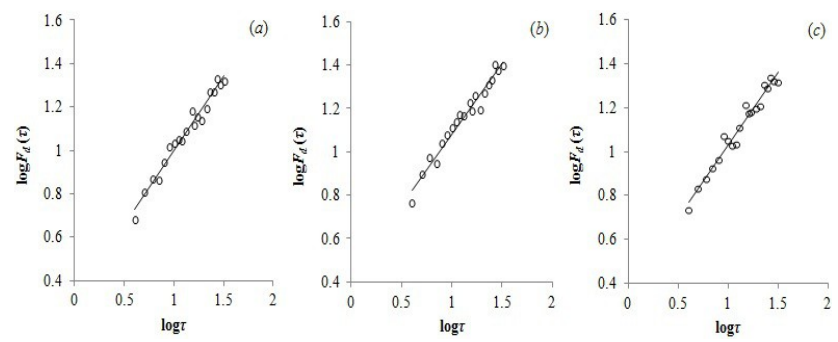

Fig. 3. Double logarithmic plot (10-base logarithms) of the rootmean-square fluctuation function $F_{d}(\tau)$ versus time interval $\tau$ (in years) for the detrended annual mean LSAT anomalies set (a) globally with the best fit equation ( $y=0.68 x+0.32$ with $R^{2}=0.97$ ), (b) over the $\mathrm{NH}$ with the best fit equation $(y=0.65 x+0.43$ with $\left.R^{2}=0.97\right)$, and (c) over the SH with the best fit equation ( $y=$ $0.65 x+0.37$ with $\left.R^{2}=0.97\right)$.

tests, the dataset of the derived $a$-exponents (for each geographical area) obey Gaussian distribution at $95 \%$ confidence interval (or significance level 0.05 ) with mean value $\bar{a}=0.52$ and standard deviation $s_{a}=0.08$. Moreover, the $95 \%$ confidence interval of $\bar{a}$ is

$$
\left(\bar{a}-\frac{1.96}{\sqrt{1000}} \cdot s_{a}, \bar{a}+\frac{1.96}{\sqrt{1000}} \cdot s_{a}\right) \text { i.e., }(0.515,0.525) .
$$

Thus, according to the $t$ test, the hypothesis that the shuffled values of the detrended annual mean LSAT anomalies follow the random (white) noise $(0.5 \pm 0.04)$ is accepted at $95 \%$ confidence interval. As an example, Fig. 4 shows the DFA results of one of the random samplings with $a=0.51$ and standard deviation 0.03 for each geographical area. In other words, we find that the DFA exponent that characterizes the fluctuations in the shuffled data is near 0.5 , thus indicating that the Joseph phenomenon is the main effect (Mandelbrot and Wallis, 1968; Rybski et al., 2008). On the other hand, the estimated DFA exponents depicted in Fig. 3 (i.e., $0.68 \pm 0.03$ and $0.65 \pm 0.03$ ) are obviously higher than the white noise- $a$, indicating that the fluctuations of the detrended annual LSAT anomalies time series exhibit power-law persistence.

The power-law persistence assumed above for the structural profile of LSAT anomalies signifies its impacts to the climate system. We emphasize that the reliably modelled values of LSAT anomalies versus time period (i.e., past and future simulations) must exhibit the same scaling properties with that existing in the real observations of the LSAT anomalies during the covered period (1880-2012). Thus, the assumed scaling property may serve as a unique criterion of the reliability of a proposed climate model. In this regard, Govindan et al. (2002) carried out a test for the scaling performance of seven leading global climate models by analysing the temperature records of six representative sites around the globe simulated by the models. The test showed that the simulated records fail to reproduce the scaling behaviour of the observed records. Consequently, the aforementioned scaling found for the temporal evolution of the 

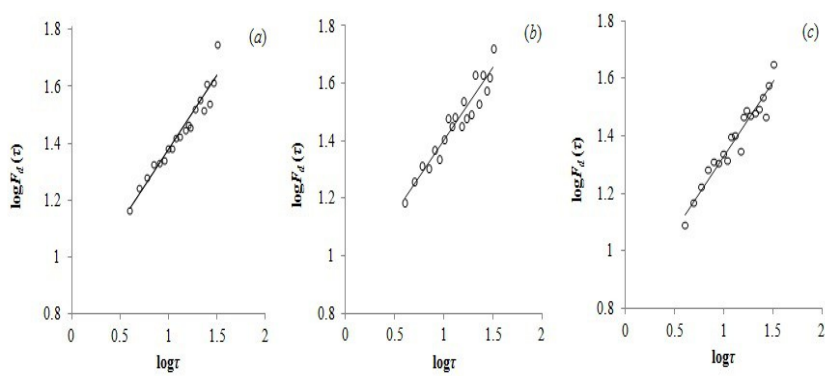

Fig. 4. Log-log plot (10-base logarithms) of the root-mean-square fluctuation function $F_{d}(\tau)$ versus time interval $\tau$ (in years) for the shuffled annual mean LSAT anomalies dataset (a) globally with the best fit equation $\left(y=0.51 x+0.86\right.$ with $\left.R^{2}=0.94\right)$, (b) over the $\mathrm{NH}$ with the best fit equation $\left(y=0.51 x+0.89\right.$ with $\left.R^{2}=0.92\right)$, and (c) over the $\mathrm{SH}$ with the best fit equation $(y=0.51 x+0.82$ with $\left.R^{2}=0.93\right)$.

surface air temperature over land of both hemispheres or globally must not be violated by the global climate models. It is important to note here that the inference of long-range correlations (or long-term memory) from the above-mentioned DFA results, prerequisites that the power-law scaling needs to be established, and a simple exponential decay of the autocorrelation function at long time scales have to be rejected. In relation to this, Maraun et al. (2004) reported verbatim: "The main insight is, that power-law scaling of the fluctuation function and thus long-memory may not be assumed a priori but have to be established." With regard to the rejection of the exponential decay of the autocorrelation function, Maraun et al. (2004) confirmed the earlier finding of Taqqu et al. (1995) and concluded verbatim: "For time series with algebraically decaying autocorrelations it can be shown that in the limit of large scales the fluctuation function increases according to a power law (Taqqu et al., 1995)." Both prerequisites will be analysed in the next sub-sections.

\subsection{DFA exponent in the time series of the monthly mean LSAT anomalies}

In order to confirm the above discussed results, we also analysed the monthly mean values of LSAT anomalies covering the whole Earth and both hemispheres, during the same period 1880-2011.

Firstly, the long-term trend was excluded by calculating the departures of the monthly mean values of LSAT anomalies from the polynomial best fit (6th degree) to the whole LSAT anomalies time series, over the whole Earth and both hemispheres, separately.

Secondly, the analysis of the time series of the detrended monthly mean LSAT anomalies started by asking if the LSAT anomaly at a given instant has any correlation with the LSAT anomaly in a later time, i.e., if the LSAT time series exhibits long-range correlations. However, this presupposes the rejection of a simple exponential decay of the autocorrelation

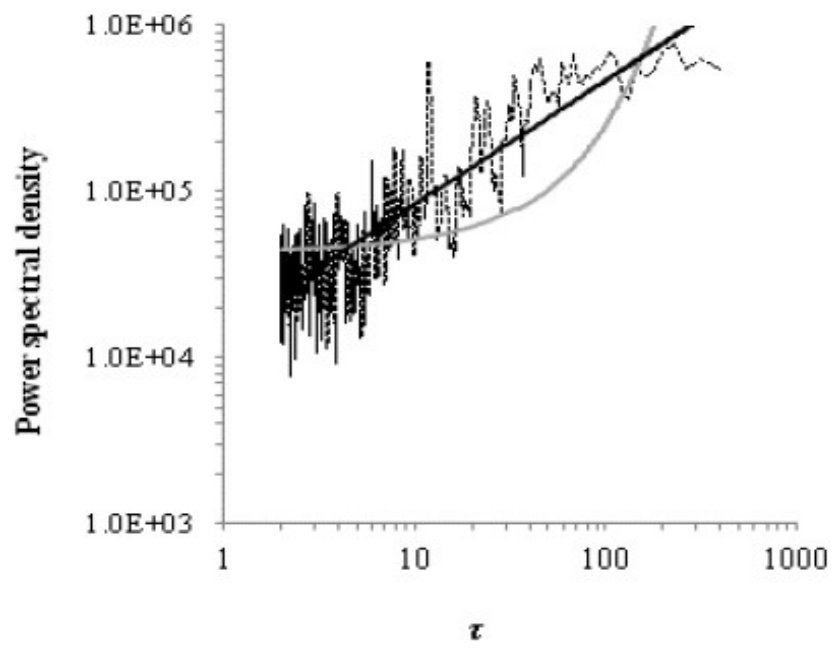

Fig. 5. Power spectral density of the detrended monthly mean LSAT anomalies (over the whole Earth) with the power-law (black line) and the exponential (grey line) fit $\left(y=1499 x^{0.75}\right.$ with $R^{2}=0.75$ and $y=42963 \mathrm{e}^{0.02 x}$ with $R^{2}=0.32$, respectively).
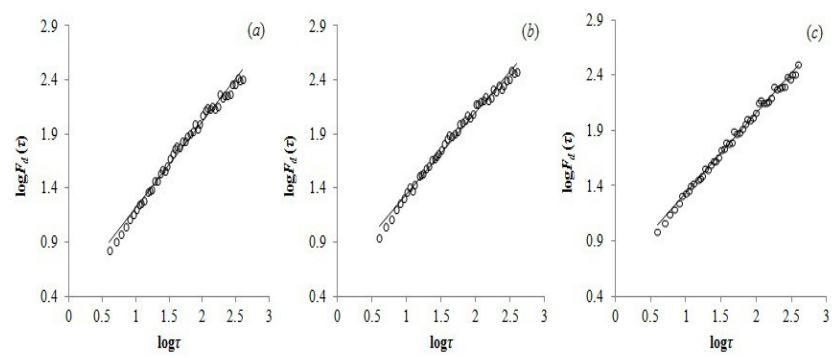

Fig. 6. Log-log plot (10-base logarithms) of the root-mean-square fluctuation function $F_{d}(\tau)$ versus time interval $\tau$ (in months) for the detrended monthly mean LSAT anomalies dataset (a) globally with the best fit equation ( $y=0.80 x+0.41$ with $\left.R^{2}=0.99\right)$, (b) over the NH with the best fit equation $\left(y=0.75 x+0.60\right.$ with $\left.R^{2}=0.99\right)$, and (c) over the SH with the best fit equation $(y=0.73 x+0.60$ with $\left.R^{2}=0.99\right)$. Note: By removing the cross-over point that corresponds to around 50 months, the DFA exponent in (a) slightly decreases $(=0.78 \pm 0.01)$ and in $(\mathbf{b})$ remains the same.

function. In this regard, the profile of the power spectral density of the detrended monthly mean LSAT anomalies over the globe, shown in Fig. 5, is better fitted algebraically (by a power law) than by an exponential function, a fact that is certified by the $F$ test at the $95 \%$ confidence interval.

Thirdly, the DFA exponents obtained from the analysis of the detrended time series of the monthly mean LSAT anomalies over both hemispheres are almost equal $(0.75 \pm 0.01$ and $0.73 \pm 0.01$ for the $\mathrm{NH}$ and $\mathrm{SH}$, respectively) approaching that of the global LSAT anomalies ( $a=0.80 \pm 0.01)$ (Fig. 6). This result supports the equal contribution of the land to the scaling of the surface air temperature over both hemispheres. Moreover, the fluctuations of the monthly means of the LSAT anomalies reveal persistent power law for scales up to $32 \mathrm{yr}$. 
It should be noted that the "cross over" shown in Fig. 6a, b at $\log \tau=1.7$ (which corresponds to $\tau=50$ months) stems from the insignificant El Niño-southern oscillation (ENSO) signal into the time series (Chen et al., 2002). Excluding this ENSO signal with the application of Wiener filtering, the DFA exponent in Fig. 6a slightly decreases, becoming $a=0.78 \pm 0.01$, while it does not change in Fig. 6b. Remarkably, such a crossover at Fig. 6c (i.e., SH) was not observed.

As mentioned in the Introduction, Efstathiou et al. (2011) suggested that the monthly mean LSST anomalies data during the period January 1850-August 2008 obey persistent power-law correlations for long scales up to $39 \mathrm{yr}$, which are stronger at the SH. In more detail, Efstathiou et al. (2011) have found that the LSST anomalies scaling exponents are $a=0.78 \pm 0.01$ and $a=0.89 \pm 0.02$ in the NH and SH, respectively. This is a composite outcome of the air temperature persistence over both land and sea, suggesting that the air temperature of the $\mathrm{SH}$ behaves more persistently. The reason, however, has not been explained so far.

The analysis presented here stresses that the scaling exponents of monthly mean LSAT anomalies for NH and SH are almost equal ( $a=0.75 \pm 0.01$ and $a=0.73 \pm 0.02$, respectively). It clearly suggests that the land contributes equally to the scaling of the surface air temperature of both hemispheres, independently of their peculiarities (e.g., $\mathrm{NH}, \mathrm{SH}$ land mass ratio and albedo). Thus, the net result drawn is that the difference between the scaling exponents in both sea and land contributions to the surface air temperature stems mainly from the sea surface temperature (SST), which exhibits stronger scaling in the Southern than in the Northern Hemisphere. This finding is in line with that of Monetti et al. (2003), according to which a stronger persistence is observed over the oceans (Atlantic and Pacifc Oceans) than over the continents. Scaling in SST up to decades was also demonstrated in observations and coupled atmosphereocean models with complex and mixed-layer oceans by Fraedrich and Blender (2003) and others (e.g., Ausloos and Ivanova, 2001; Eichner et al., 2003). From a physical point of view, the strong tendency of the air temperature over ocean at a particular period to remain the same during the next period (strong persistence) is expected, because the oceans have the greatest capacity to store heat (covering almost three quarters of the Earth's surface), thus being able to regulate the temperature on land. The latter may probably be the reason for the less pronounced persistence of temperature over land.

It can be argued that simple correlations between SST and LSAT or LSST anomalies over NH and SH could shed light on the aforementioned difference in DFA exponents. In general, however, it is not straightforward that two time series that are significantly correlated will lead to the same DFA exponents. In addition, it should be recalled that the local changes of SST influences water vapour and clouds (through the activation of thermodynamical processes). However, water vapour and clouds are sensitive to many other factors that may depend only partly on the local SST (e.g., the large-scale atmospheric circulation, which transports heat and moisture affecting the thermodynamical stability of the atmosphere on large spatial scales). In this context, positive correlations between SST, water vapour, and clear-sky greenhouse effect have been interpreted as manifestations of the positive water vapour feedback (Bony et al., 1997; Raval and Ramanathan, 1989).

\subsection{DFA - local slopes in the time series of the annual and monthly mean LSAT anomalies}

As discussed above (Sect. 2), Franzke et al. (2012) suggested that the two phenomena of LRD and non-Gaussian behaviour commonly occur together in a complex system, discussing, in particular, two models which can simultaneously account for both phenomena: autoregressive fractional integrated moving average (ARFIMA) and linear fractional stable motion (LFSM).

Keeping this in mind, we checked the time series used in our paper for Gaussian behaviour by employing the Kolmogorov-Smirnov and Anderson-Darling best fit tests. The outcome is that the detrended annual and monthly mean LSAT anomalies time series obey a Gaussian distribution at the $95 \%$ confidence interval.

As we said above, another point which needs further exploration is pointed out by Maraun et al. (2004): "To reliably infer power-law scaling, it has to be established and not be assumed. This can be done by estimating local slopes and investigating them for constancy in a sufficient range." Therefore, since the single straight line of the DFA plot for the detrended and deseasonalised (by Wiener filtering) monthly mean global LSAT anomalies established in the whole range of scales may be biased, we evaluated the local slopes of $\log F_{d}(\tau)$ vs. $\log \tau$, detecting for constancy in a sufficient range. Along these lines, we first fitted a straight line to $\log F_{d}(\tau)$ vs. $\log \tau$ within a small window. This window was then shifted successively over all calculated scales $\tau$. Figure 7 illustrates the local slopes $a$ vs. $\log \tau$ for four different window sizes. Inspection of Fig. 7 shows that for small scales the variance of the local slopes is low, while for large scales the variance is increased. Moreover, the local slope $a$ seems to be fluctuated around a constant value $(a \approx 0.78)$ at the range 3-16yr, approximately. In addition, we computed the standard deviations, $s_{a}$, of each estimated local slope over all the scales considered, separately for both window sizes, and the error bounds of each local slope $a$ that were defined as $a \pm 1.96 \cdot s_{a}$ (see Fig. 7).

In the following, we applied the non-parametric WaldWolfowitz statistical hypothesis test (Wald and Wolfowitz, 1940) in order to examine the hypothesis $H_{o}$ that the distribution of the local slopes for the detrended and deseasonalised monthly mean global LSAT anomalies could be considered identical to the distribution of the local slopes for a simulated white noise time series. As it was derived, the hypothesis $H_{o}$ is rejected at $95 \%$ confidence interval, using 


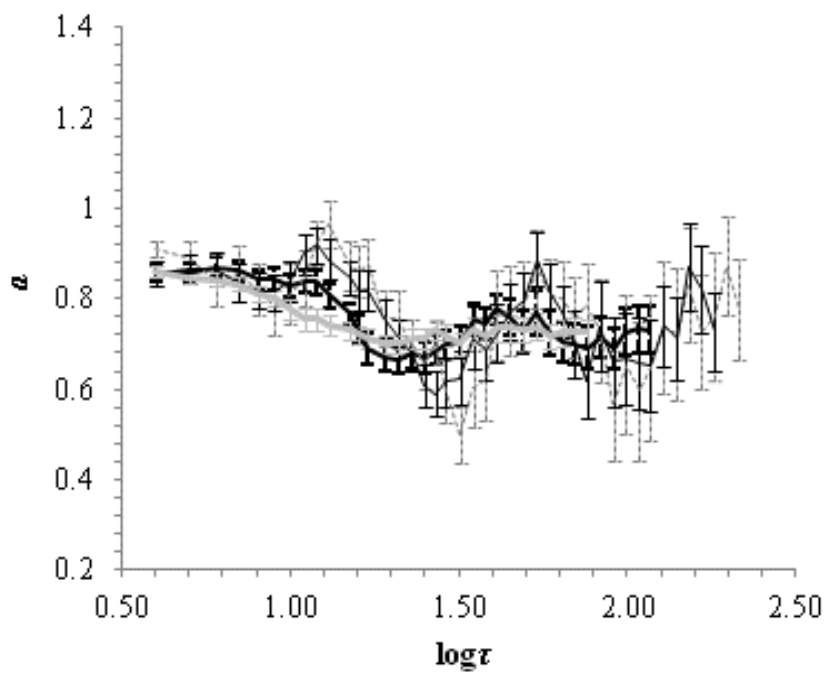

Fig. 7. Local slopes of the $\log F_{d}(\tau)$ vs. $\log \tau$ (10-base logarithms) calculated within a window of 8 points (dashed grey line), of 10 points (solid thin black line), of 15 points (solid bold black line) and of 20 points (solid bold grey line) for the detrended time series of the mean monthly global LSAT anomalies. The grey and black error bars indicate the corresponding $1.96 \cdot s_{a}$-intervals of the slopes over all the considered scales.

each of the windows (of $8,10,15$ and 20 points) separately. Furthermore, we calculated the weighted mean of the local slopes $a$ using the inverse of their standard deviations $1 / s_{a}$ as weights; the derived value 0.79 seemed not to belong in the $2 s$-interval of the Monte Carlo mean value $\bar{a}=0.52$. Inspection of Fig. 7, regarding the bias and variability of the estimated local slopes $a$ vs. $\log \tau$, reveals all the features of a long-memory process and differs adequately from a shortrange scaling behaviour (Maraun et al., 2004).

Next, the above mentioned analysis was repeated for the case of the NH. In more detail, Fig. 8 depicts the results obtained when applying the DFA-local slopes method on the detrended and deseasonalised mean monthly data of the $\mathrm{NH}$. It is clearly seen from Fig. 8 that for large scales $(3-16 \mathrm{yr})$ the local slope is fluctuating around a constant value $(a \approx 0.75$, thus establishing the power-law scaling. The method of local slopes was also applied to the mean monthly data of the $\mathrm{SH}$, giving similar results to the above. Additionally, we applied the method of the DFA-local slopes to the annual data of the global LSAT anomalies for two different window sizes. The result obtained was that the local slope $a$ vs. $\log \tau$ is fluctuates around a constant value $(\sim 0.68)$. Moreover, a Wilcoxon test showed that the mean value of the local slopes for the large scales is different from 0.5 at $95 \%$ confidence interval. The same method was also applied to the annual data of the $\mathrm{NH}$ and $\mathrm{SH}$ providing same results.

Finally, in order to clarify whether the annual and the monthly mean time series reveal nearly equal scaling, we applied the non-parametric Wald-Wolfowitz statistical test.

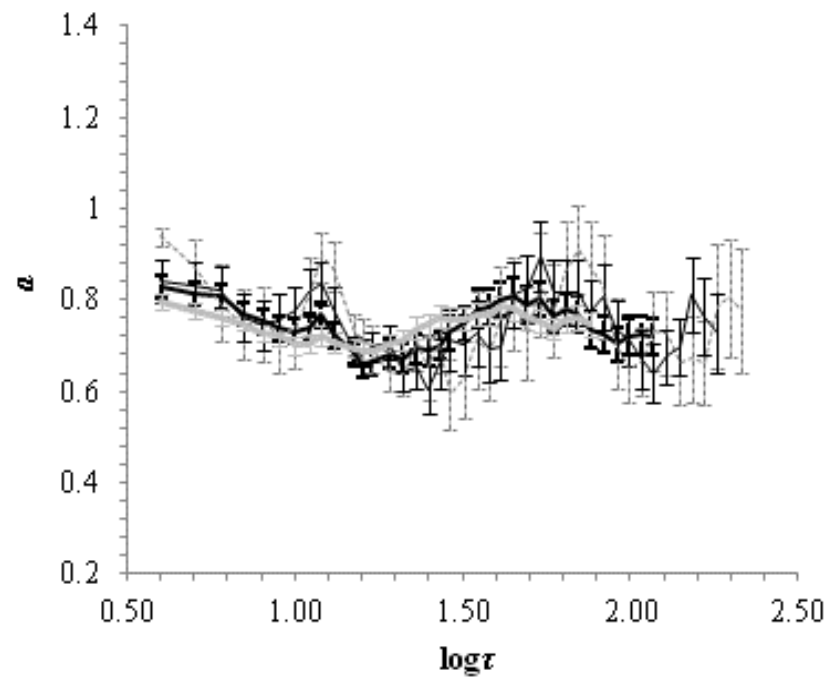

Fig. 8. As in Fig. 7, but for the detrended and deseasonalised time series of the mean monthly LSAT anomalies of the Northern Hemisphere.

We attempted to examine the hypothesis $H_{o}$ that the mean value of the local slopes for the detrended annual mean LSAT anomalies time series could be considered as equal to the mean value of the local slopes for the detrended monthly mean LSAT anomalies time series (over whole Earth, NH and $\mathrm{SH}$, separately). As it is derived, the hypothesis $H_{o}$ cannot be rejected at the $95 \%$ confidence interval, using indicatively the window of 7 (8) points for the annual (monthly) data. Besides, Xu et al. (2010), studying coarse-graining in time where data are averaged in non-overlapping time windows, have reached the conclusion that the scaling curve $F(n)$ shifts down due to the decrease in the standard deviation of the coarse-grained signal, and that the scaling is practically preserved for positively correlated signals, while for anti-correlated signals there is a slight decrease in the scaling exponent. For instance, in Fig.3a (where the annual mean global LSAT anomalies set is analysed), $\log \tau<1.5$ (i.e., $\tau<31.6 \mathrm{yr}$ ) and in Fig. 6a (where the monthly mean global LSAT anomalies set is analysed), $\log \tau<2.58$ (i.e., $\tau<380.2$ months $=31.6 \mathrm{yr}$ ), thus confirming that the persistent scaling is practically preserved.

\subsection{DFA exponent in the time series of the annual mean LSAT anomalies versus latitude}

In the following, we studied the annual mean values of LSAT anomalies over selected latitude zones, during the period 1880-2011. Table 1 depicts the DFA value for the polynomially detrended time series of the annual mean LSAT anomalies versus latitude. The obtained $a$-exponent ranges between $(0.51,0.79)$ with standard deviation 0.03 . These DFA exponents point to power-law scaling, which, after the rejection of the exponential decay of the autocorrelation functions 
Table 1. DFA-exponent for the annual mean LSAT anomalies versus latitude.

\begin{tabular}{lllll}
\hline NH Latitude & Equator-24 $4^{\circ} \mathrm{N}$ & $24^{\circ} \mathrm{N}-44^{\circ} \mathrm{N}$ & $44^{\circ} \mathrm{N}-64^{\circ} \mathrm{N}$ & $24^{\circ} \mathrm{N}-90^{\circ} \mathrm{N}$ \\
\hline$a$-value & $0.52 \pm 0.02$ & $0.55 \pm 0.03$ & $0.6 \pm 0.02$ & $0.63 \pm 0.03$ \\
SH Latitude & Equator $-24^{\circ} \mathrm{S}$ & $24^{\circ} \mathrm{S}-44^{\circ} \mathrm{S}$ & $44^{\circ} \mathrm{S}-64^{\circ} \mathrm{S}$ & $24^{\circ} \mathrm{S}-90^{\circ} \mathrm{S}$ \\
$a$-value & $0.51 \pm 0.02$ & $0.74 \pm 0.03$ & $0.76 \pm 0.02$ & $0.79 \pm 0.03$ \\
\hline
\end{tabular}

for large scales and the investigation of the local slopes of the fluctuation functions, imply long-range persistence over the latitude zones $24^{\circ} \mathrm{N}-44^{\circ} \mathrm{N}, 24^{\circ} \mathrm{S}-44^{\circ} \mathrm{S}, 44^{\circ} \mathrm{N}-64^{\circ} \mathrm{N}$, $44^{\circ} \mathrm{S}-64^{\circ} \mathrm{S}, 24^{\circ} \mathrm{N}-90^{\circ} \mathrm{N}, 24^{\circ} \mathrm{S}-90^{\circ} \mathrm{S}$ and the classical random walk (white noise) over the zones Equator- $24^{\circ} \mathrm{N}$ and Equator-24 $\mathrm{S}$.

The poleward increase of the power-law exponents of the annual mean values of the LSAT anomalies in both hemispheres could be associated with the poleward increase in climate sensitivity predicted by global climate models. Nevertheless, the plausible reasons for the classical random walk over the tropics need further elaboration.

With regard to the poleward increase in climate sensitivity, several studies considering slow and fast feedbacks have shown that climate sensitivity depends upon several climate parameters (e.g., Varotsos, 2002). In particular, climate sensitivity is closely associated with the levels of GHGs, albedo, ice and snow, sea-ice coverage and thickness, moisture transfer, heat transported by the atmosphere and/or ocean, cloudiness, etc. The sum of these feedbacks yields net positive radiative forcing at the surface in high latitudes (e.g., Govindasamy et al., 2005; Holland and Bitz, 2003; Manabe and Stouffer, 1980; Pagani et al., 2010). As an example, on a warming Earth more energy is transported to the poles because the water vapour and cloud feedbacks are strongly positive at low latitudes and decrease drastically with increasing latitude, thus generating an equator-to-pole gradient. In this regard, Zelinka and Hartmann (2012) found that reduced heat flux from the high-latitude ocean further amplifies the equator-to-pole gradient in atmospheric energy loss, which requires the atmosphere to increase its share of the total poleward energy transport.

\section{Conclusions}

We applied the detrended fluctuation analysis to the annual and the monthly mean values of the LSAT anomalies over the globe and both hemispheres for the period 1880-2011 in order to search for intrinsic dynamical properties.

According to the analysis and discussion presented above, the following conclusions may be drawn:

1. The annual and monthly mean LSAT anomalies time series, all over the globe and both hemispheres, exhibit persistent power-law scaling. To confirm this result the autocorrelation function and the method of the local slopes of the fluctuation functions were employed. The results obtained show a rejection of an exponential decay of the autocorrelation function for large scales and a constancy of the local slopes in a sufficient range. Both results, along with the calculated error estimates, confirm the existence of LRD in the LSAT anomalies.

2. The scaling exponents of the annual (monthly) mean LSAT anomalies, $a=0.65(0.73-0.75), \quad$ are roughly equal in both hemispheres, approaching that of the global annual (monthly) mean LSAT anomalies, $a=0.68$ (0.80), respectively. Comparing this result with our earlier finding (Efstathiou et al., 2011) that the LSST anomalies obey stronger scaling exponent $(a=0.89)$ in the SH than in the NH $(\alpha=0.78)$, we reach the conclusion that the main contribution of the difference between the scaling exponents in both sea and land to the surface air temperature stems mainly from the SST, which exhibits stronger scaling in the SH than in the NH. This conclusion is in accordance with the basic knowledge that oceans have the greatest capacity to store heat, being thus able to regulate the temperature on land with less pronounced persistence.

3. The annual mean values of LSAT anomalies over the latitude zones $24^{\circ} \mathrm{N}-44^{\circ} \mathrm{N}, 24^{\circ} \mathrm{S}-44^{\circ} \mathrm{S}, 44^{\circ} \mathrm{N}-64^{\circ} \mathrm{N}$, $44^{\circ} \mathrm{S}-64^{\circ} \mathrm{S}, 24^{\circ} \mathrm{N}-90^{\circ} \mathrm{N}, 24^{\circ} \mathrm{S}-90^{\circ} \mathrm{S}$ exhibit persistent power-law scaling, with gradually increasing scaling exponents with latitude and more pronounced over SH. The investigation of the local slopes of the fluctuation functions revealed the reliability of the scaling effect and the persistent long-range correlations. Remarkably, the tropical region (Equator-24 ${ }^{\circ} \mathrm{N}$ and Equator$24^{\circ} \mathrm{S}$ ) obeys the classical random walk (white noise).

It should be emphasized that while it can never be ruled out that a process is not truly scaling, the analysis presented above for LSAT provides robust evidence for scaling behaviour over almost two decades (i.e., constancy of local slopes at long time scales), and that a power law fits the spectrum better than an exponential function. These two findings provide reasonably strong evidence for scaling behaviour in LSAT, which is a new finding in the scientific literature. As pointed out by Maraun et al. (2004), there have been several attempts in the past to detect long memory at various climatic parameters using the DFA tool, but in most cases without scrutinizing the sensitivity (i.e., compatibility with 
LRD, whensoever present) and specificity (rejection of LRD, when not present) of the DFA algorithm. In our case, the DFA algorithm exhibited appropriate sensitivity and specificity. In addition, long-range correlations in the climate system have been considered sometimes as recurrences or periodicities (e.g., the Milankovitch-cycles). However, as mentioned in the Introduction, the concept of long-memory refers to non-periodic processes even if their (deterministic) behaviour generates correlations for infinite time lags (Maraun et al. 2004; Markonis and Koutsoyiannis, 2013).

The observed poleward stronger persistence in LSAT could be in general a result of either stronger positive feedbacks or larger inertia. In addition, the scaling property detected in the in-field observations of the LSAT anomalies could serve as a test for the state-of-the-art and the scaling performance of the advanced global climate models for confident projections of climate change (e.g., Theme Issue, Thompson and Sieber, 2012).

Acknowledgements. We wish to thank both reviewers for their fruitful comments.

Edited by: P. Haynes

\section{References}

Alvarez-Ramirez, J., Alvarez, J., Dagdug, L., Rodriguez, E., and Echeverria, J. C.: Long-term memory dynamics of continental and oceanic monthly temperatures in the recent 125 years, Physica A 387, 3629-3640, 2008.

Anderson, T. W. and Darling, D. A.: A Test of Goodness-of-Fit, J. Am. Stat. Assoc., 49, 765-769, 1954.

Alexandris, D., Varotsos, C., Kondratyev, K. Y. and Chronopoulos, G.: On the altitude dependence of solar effective UV, Phys. Chem. Earth Part C-Sol.-Ter. Planet. Sci., 24, 515-517, 1999.

Anthis, A. I. and Cracknell, A. P.: A method for classification accuracy evaluation for a precipitation-oriented classifier, Int. J. Rem. Sens., 26, 4785-4800, 2005.

Audit, B., Bacry, E., Muzy, J. F. and Arneodo, A.: Wavelets based estimators of scaling behavior, IEEE Trans Inform. Theory, 48, 2938-2954, 2002.

Ausloos, M. Ivanova, K.: Power-law correlations in the southern - oscillation - index fluctuations characterizing El Nino, Phys. Rev. E, 63, 047201, doi:10.1103/PhysRevE.63.047201, 2001.

Bony, S., Lau, K.-M., and Sud, Y. C: Sea Surface Temperature and Large-Scale Circulation Influences on Tropical Greenhouse Effect and Cloud Radiative Forcing. J. Climate, 10, 2055-2077, 1997.

Bryce, R. M. and Sprague, K. B.: Revisiting detrended fluctuation analysis, Sci. Rep., 2, 1-6, doi:10.1038/srep00315, 2012.

Chattopadhyay, S. and Chattopadhyay, G.: Univariate modelling of summer-monsoon rainfall time series: comparison between ARIMA and ARNN, Compt. Rend. Geosci., 342, 100-107, 2010.

Chandra, S. and Varotsos, C. A.: Recent trends of the total column ozone - implications for the Mediterranean region, Int. J. Remote Sens., 16, 1765-1769, 1995.
Chen, Z., Ivanov, P., Hu, K., and Stanley, H.: Effect of nonstationarities on detrended fluctuation analysis, Phys. Rev. E, 65, 041107, 1-15, 2002.

Coronado, A. V. and Carpena, P.: Size effects on correlation measures, J. Biolog. Phys. 31, 121-133, doi:10.1007/s10867-0053126-8C, 2005.

Delignieres, D., Ramdani, S., Lemoine, L., Torre, K., Fortes, M., and Ninot, G.: Fractal analyses for 'short' time series: A reassessment of classical methods. J. Math. Psychol., 50, 525-544, 2006.

Efstathiou, M. N. and Varotsos, C.: On the altitude dependence of the temperature scaling behavior at the global troposphere, Int. J. Remote Sens., 31, 343-349, 2010.

Efstathiou, M. N., Varotsos, C., and Kondratyev, K. Y.: An estimation of the surface solar ultraviolet irradiance during an extreme total ozone minimum, Meteorol. Atmos. Phys., 68, 171176, 1998.

Efstathiou, M. N., Tzanis C., Cracknell A. and Varotsos C. A.: New features of the land and sea surface temperature anomalies, Int. J. Remote Sens., 32, 3231-3238, 2011.

Efstathiou, M. N., Varotsos, C., Singh, R. P., Cracknell, A. P., and Tzanis, C.: On the longitude dependence of total ozone trends over middle-latitudes, Int. J. Remote Sens., 24, 13611367, 2003.

Eichner, J. F., Koscielny-Bunde, E., Bunde, A., Havlin, S., and Schellnhuber, H. J.: Power-law persistence and trends in the atmosphere: A detailed study of long temperature records, Phys. Rev. E, 68, 046133, doi:10.1103/PhysRevE.68.046133, 2003.

Feretis, E., Theodorakopoulos, P., Varotsos, C., Efstathiou, M., Tzanis, C., Xirou, T., Alexandridou, N., and Aggelou, M.: On the plausible association between environmental conditions and human eye damage, Environ. Sci. Pollut. R., 9, 163-165, 2002.

Fraedrich, K. and Blender, R.: Scaling of Atmosphere and Ocean Temperature Correlations in Observations and Climate Models, Phys. Rev. Lett., 90, doi:10.1103/PhysRevLett.90.108501, 2003.

Franzke, C. L. E., Graves, T., Watkins, N. W., Gramacy, R. B., and Hughes, C.: Robustness of estimators of long-range dependence and self-similarity under non-Gaussianity, Phil. Trans. R. Soc. A, 370, 1250-1267, 2012.

Gerber, E. P., Butler, A., Calvo, N., Charlton-Perez, A., Giorgetta, M. and co-authors.: Assessing and understanding the impact of stratospheric dynamics and variability on the earth system, B. Am. Meteorol. Soc., 93, 845-859, doi:10.1175/BAMS-D-1100145.1, 2012.

Geweke, J. and Porter-Hudak, S.: The estimation and application of long memory time series models, J. Time Series Anal., 4, 221 238, 1983.

Govindasamy, B., Thompson, S., Mirin, A., Wickett, M., Caldeira, K., and Delire, C.: Increase of carbon cycle feedback with climate sensitivity: results from a coupled climate and carbon cycle model, Tellus B, 57, 153-163, 2005.

Govindan, R. B., Vyushin, D., Bunde, A., Brenner, S., Havlin, S., and Schellnhuber, H. J.: Global climate models violate scaling of the observed atmospheric variability, Phys. Rev. Let., 89, 028501, 1139-1159, 2002.

Hausdorff, J. M. and Peng, C.-K.: Multiscaled randomness: A possible source of 1/f noise in biology, Phys. Rev. E, 54, 2154-2157, 1996. 
Holland, M. M. and Bitz, C. M.: Polar ampli?cation of climate change in coupled models, Clim. Dynam., 21, 221-232, 2003.

Hurvich, C. M. and Brodsky, J.: Broadband Semiparametric Estimation of the Memory Parameter of a Long-Memory Time Series Using Fractional Exponential Models, J. Time Anal., 22, 221249, 2001

Kantelhardt, J. W., Zschiegner, S. A., Koscielny-Bunde, E., Havlin, S., Bunde, A., and Stanley, H. E.: Multifractal detrended fluctuation analysis of nonstationary time series, Phys. A, 316, 87-114, 2002.

Katsambas, A., Varotsos, C. A., Veziryianni, G., and Antoniou, C.: Surface solar ultraviolet radiation: A theoretical approach of the SUVR reaching the ground in Athens, Greece, Environ. Sci. Pollut. Res., 4, 69-73, 1997.

Kondratyev, K. Y. and Varotsos C.: Atmospheric greenhouse effect in the context of global climate-change, Nuovo Cim. Soc. Ital. Fisica C - Geophys. Space Phys., 18, 123-151, 1995a.

Kondratyev, K. Y. and Varotsos, C. A.: Volcanic eruptions and global ozone dynamics, Int. J. Remote Sens., 16, 1887-1895., 1995b.

Kondratyev, K. Y. and Varotsos, C. A.: Atmospheric ozone variability in the context of global change, Int. J. Remote Sens., 16, 1851-1881, 1995c.

Kondratyev, K. Y. and Varotsos, C. A.: Global total ozone dynamics - Impact on surface solar ultraviolet radiation variability and ecosystems, Environ. Sci. Pollut. Res, 3, 205-209, 1996.

Koscielny-Bunde, E., Bunde, A., Havlin, S., Roman, H. R., Goldreich, Y., and Schellnhuber, H. J.: Indication of a Universal Persistence Law Governing Atmospheric Variability, Phys. Rev. Lett., 81, 729-732, 1998.

Koutsoyiannis, D., Efstratiadis, A., Mamassis, N., and Christofides, A.: On the credibility of climate predictions, Hydrolog. Sci. J., 53, 671-684, 2008.

Ludecke, H.-J., Link, R., and Ewart, F.-K.: How Natural is the Recent Centennial Warming? An Analysis of 2249 Surface Temperature Records, Int. J. Mod. Phys. C, 22, doi:10.1142/S0129183111016798, 2011.

Markonis, Y. and Koutsoyiannis, D.: Climatic Variability Over Time Scales Spanning Nine Orders of Magnitude: Connecting Milankovitch Cycles with Hurst-Kolmogorov Dynamics, Surv. Geophys., 34, 181-207, 2013.

Manabe, S. and Stouffer, R. J.: Sensitivity of a global climate model to an increase of $\mathrm{CO}_{2}$ concentration in the atmosphere, J. Geophys. Res., 85, 5529-5554, 1980.

Mandelbrot, B. B. and Wallis, J. R.: Noah, Joseph, and operational hydrology, Water Resour. Res., 4, 909-918, 1968.

Maraun, D., Rust, H. W., and Timmer, J.: Tempting long-memory on the interpretation of DFA results, Nonlinear Proc. Geoph., 11, 495-503, 2004.

Melnikova, I.: Comparative assessment of the impact of molecular scattering and total ozone content on the characteristics of UV radiation in the atmosphere, Int. J. Remote Sens., 30, 6141-6150, 2009.

Monetti, R. A., Havlin, S., and Bunde, A.: Long-term persistence in the sea surface temperature fluctuations, Phys. A, 320, 581-589, 2003.

Pagani, M., Liu, Z., LaRiviere, J., and Ravelo, A. C.: High Earthsystem climate sensitivity determined from Pliocene carbon dioxide concentrations, Nature Geosci., 3, 27-30, 2010.
Peng, C.-K., Buldyrev, S. V., Havlin, S., Simons, M., Stanley, H. E., and Goldberger, A. L.: Mosaic organization of DNA nucleotides, Phys. Rev. E, 49, 1685-1689, 1994.

Raval, A. and Ramanathan, V.: Observational determination of the greenhouse effect, Nature, 342, 758-761, 1989.

Rust, H. W.: Detection of long-range dependence - applications in climatology and hydrology, Ph.D. thesis, Potsdam University, Potsdam, Germany, 2007.

Rybski, D., Bunde, A., Havlin, S., and von Storch, H.: Long-term persistence in climate and the detection problem, Geophys. Res. Let., 33, 106718, doi:10.1029/2005gl025591, 2006.

Rybski, D., Bunde, A., Havlin, S., and von Storch, H.: Long-term memory in 1000-year simulated temperature records, J. Geophys. Res., 113, D02106, doi:10.1029/2007JD008568, 2008.

Stephens, M. A.: EDF Statistics for Goodness of Fit and Some Comparisons. J. Am. Stat. Assoc., 69, 730-737, 1974.

Taqqu, M. S., Teverovsky, V., and Willinger, W.: Estimators for long-range dependence: An empirical study, Fractals, 3, 785798, 1995.

Thompson, J. M. and Sieber, J.: Climate predictions: the influence of nonlinearity and randomness, Phil. Trans. R. Soc. A, 370, 1007-1011, doi:10.1098/rsta.2011.0423, 2012.

Tsonis, A. and Elsner, J.: Testing for Scaling in Natural Forms and Observables, J. Stat. Phys., 81, 869-880, 1995.

Tzanis, C. and Varotsos, C. A.: Tropospheric aerosol forcing of climate: a case study for the greater area of Greece, Int. J. Remote Sens., 29, 2507-2517, 2008.

Varotsos, C.: The southern hemisphere ozone hole split in 2002, Environ. Sci. Pollut. Res., 9, 375-376, 2002.

Varotsos, C.: Power-law correlations in column ozone over Antarctica, Int. J. Rem. Sens., 26, 3333-3342, 2005a.

Varotsos, C.: Modern computational techniques for environmental data; Application to the global ozone layer, (Proceedings of the ICCS 2005) Lecture Notes in Computer Science, 3516, 504-510, $2005 b$.

Varotsos, C. and Kirk-Davidoff, D.: Long-memory processes in ozone and temperature variations at the region $60^{\circ} \mathrm{S}-60^{\circ} \mathrm{N}$, Atmos. Chem. Phys., 6, 4093-4100, doi:10.5194/acp-6-4093-2006, 2006.

Varotsos, C., Kalabokas, P., and Chronopoulos, G.: Association of the laminated vertical ozone structure with the lower stratospheric circulation, J. Appl. Meteorol., 33, 473-476, 1994.

Varotsos, C., Ondov, J., and Efstathiou, M.: Scaling properties of air pollution in Athens, Greece and Baltimore, Maryland, Atmos. Environ., 39, 4041-4047, 2005.

Varotsos, C., Assimakopoulos, M. N. and Efstathiou, M.: Technical Note: Long-term memory effect in the atmospheric $\mathrm{CO}_{2}$ concentration at Mauna Loa, Atmos. Chem. Phys., 7, 629-634, doi:10.5194/acp-7-629-2007, 2007.

Varotsos, C. A., Ondov, J. M., Cracknell, A. P., Efstathiou, M. N., and Assimakopoulos, M. N.: Long-range persistence in global Aerosol Index dynamics, Int. J. Remote Sens., 27, 3593-3603, 2006.

Varotsos, P. A., Sarlis, N. V., and Skordas, E. S.: Long-range correlations in the electric signals that precede rupture, Phys. Rev. E, 66, 011902, doi:10.1103/PhysRevE.66.011902, 2002.

Varotsos, P. A., Sarlis, N. V., and Skordas, E. S.: Longrange correlations in the electric signals that precede rupture: Further investigations, Phys. Rev. E, 67, 021109, 
doi:10.1103/PhysRevE.67.021109, 2003.

Varotsos, P. A., Sarlis, N. V., and Skordas, E. S.: Detrended fluctuation analysis of the magnetic and electric field variations that precede rupture, CHAOS 19, 023114, 2009.

Wald, A. and Wolfowitz, J.: On a test whether two samples are from the same population, Ann. Math. Statist., 11, 147-162, 1940.

Weber, R. O. and Talkner, P.: Spectra and correlations of climate data from days to decades, J. Geophys. Res., 106, 20131-20144, 2001.

Xu, Y., Ma, Q. D. Y., Schmitt, D. T., Bernaola-Galvan, P., and Ivanov, P. C.: Effects of coarse-graining on the scaling behavior of long-range correlated and anti-correlated signals, arXiv:1002.3834v1 [physics.data-an] 19 February 2010.
Xue, Y., Ai, J., Wan, W., Guo, H., Li, Y., Wang, Y., Guang, J., Mei, L., and Xu, H.: Grid-enabled high-performance quantitative aerosol retrieval from remotely sensed data, Comput. Geosci., 37, 202-206, 2011.

Zelinka, M. D. and Hartmann, D. L.: Climate feedbacks and their implications for poleward energy flux changes in a warming climate, J. Climate, 25, 608-624, 2012. 